\title{
Spontaneous onset of irregular collective oscillations in heterogeneous neural networks
}

\author{
Stefano Luccioli ${ }^{1,2}$ \\ From Twentieth Annual Computational Neuroscience Meeting: CNS*2011 \\ Stockholm, Sweden. 23-28 July 2011
}

More and more interest is devoted to identify the basic mechanisms underlying the emergence of nontrivial collective behaviors of large populations of neurons, one typical instance being the irregular background activity in the cerebral cortex [1]. Though accurate mathematical models have been developed for describing the microscopic activity of single neurons and of their synapsis [2], it is convenient to use relatively simple systems [3], under the assumption that they could be able to reproduce the relevant collective phenomena exhibited by realistic systems.

In this perspective, in Ref. [4] we numerically studied a network composed of leaky integrate and fire neurons [5], a class of simple single neuron models that exhibit a periodic spiking behavior in the absence of synaptic coupling. The coupling is the result of pulses that are sent across the system. The natural heterogeneity in the network is represented by the fact that different neurons have different spiking frequencies. Moreover we consider a delay between the pulse emission and its arrival.

The main result here discussed, and reported in Ref. [4], is that, upon increasing the coupling strength of the neurons, such a kind of network exhibits a transition to a nontrivial collective dynamics.

Relevant differences divide our findings from the transition observed in the Kuramoto model (KM) [6], a paradigmatic model for synchronization phenomena, describing the dynamics of an ensemble of coupled phase oscillators.

First, the model that we considered is not chaotic and it eventually converges to a periodic orbit. Moreover the transient time needed to approach some periodic orbit grows exponentially with the number of neurons. Thus

\footnotetext{
Correspondence: stefano.luccioli@fi.isc.cnr.it

${ }^{1}$ CNR - Consiglio Nazionale delle Ricerche, Istituto dei Sistemi Complessi, I50019, Italy

Full list of author information is available at the end of the article
}

implying that for large population of neurons the relevant dynamical regime is the "transient", rather than the periodic orbit approached over astronomical time scales! This is an instance of "stable chaos" [7-9].

But the most striking difference with KM concerns the dynamical regime observed beyond the transition, in fact in this case the overall neural activity is not constant and not even simply periodically modulated, but exhibits irregular, seemingly chaotic, oscillations.

The mechanism here analyzed may contribute to the irregular background activity observed in the cerebral cortex.

\section{Author details}

${ }^{1}$ CNR - Consiglio Nazionale delle Ricerche, Istituto dei Sistemi Complessi, I50019, Italy. ${ }^{2}$ INFN, Sez. Firenze, I-50019 Sesto Fiorentino, Italy.

Published: 18 July 2011

\section{References}

1. Destexhe A, Rudolph M, Paré D: The high-conductance state of neocortical neurons in vivo. Nature Neurosci 2003, 4:739-751.

2. Gerstner W, Kistler WM: Spiking neuron models: Single neurons, populations, plasticity. Cambridge University Press; 2002.

3. Golomb G, Hansel D, Mato G: In Handbook of Biological Physics. Volume 4. Amsterdam:Elsevier Science; 2001.

4. Luccioli S, Politi A: Irregular Collective Behavior of Heterogeneous Neural Networks. Phys Rev Lett 2010, 105:1581041-1581044.

5. Politi A, Luccioli S: Network Science: Complexity in Nature and Technology. Heidelberg: Springer-Verlag; 2010.

6. Kuramoto Y: Chemical Oscillations, Waves, and Turbulence. Berlin: Springer-Verlag; 1984.

7. Politi A, Torcini A: Nonlinear dynamics and Chaos. Heidelberg: SpringerVerlag; 2010.

8. Jahnke S, Memmesheimer RM, Timme M: Stable Irregular Dynamics in Complex Neural Networks. Phys Rev Lett 2008, 100:0481021-0481024.

9. Zillmer R, Brunel N, Hansel D: Very long transients, irregular firing, and chaotic dynamics in networks of randomly connected inhibitory integrate-and-fire neurons. Phys Rev E 2009, 79:0319091-03190913.

doi:10.1186/1471-2202-12-S1-P206

Cite this article as: Luccioli: Spontaneous onset of irregular collective oscillations in heterogeneous neural networks. BMC Neuroscience 2011 12(Suppl 1):P206.
C Biomed Central

() 2011 Luccioli; licensee BioMed Central Ltd. This is an open access article distributed under the terms of the Creative Commons Attribution License (http://creativecommons.org/licenses/by/2.0), which permits unrestricted use, distribution, and reproduction in any medium, provided the original work is properly cited. 